\title{
Vorwort / Danksagung
}

Die Geschichte der DDR ist auch eine Geschichte der Dummheit, der Inkompetenz von Personen. Dass man in der SED von den Personen, die diese Partei vertreten oder verkörpern, so völlig abgesehen hat, erinnert mich an Graham Green, ,Die Macht und die Herrlichkeit, den Roman über den saufenden Priester in Mexico [...], immer betrunken, verkommen und asozial, aber er bleibt der Priester und verkörpert die Kirche. So sah ich damals auch die Funktionäre, sonst wäre es gar nicht möglich gewesen, mit den Leuten umzugehen. Viele waren primitiv, dumm, brutal, verkommen, gierig nach bürgerlichem Standard, überfordert alle. ${ }^{1}$

Über dreißig Jahre nach der Auflösung des ostdeutschen Staates scheint es beinah unvermeidbar, nicht minder energisch in das hier zitierte Urteil Heiner Müllers, des womöglich wichtigsten und einflussreichsten Intellektuellen der DDR, einzustimmen. Und dennoch, trotz aller vermeintlichen kulturellen, politisch-moralischen, und nicht zuletzt wirtschaftlichen Überlegenheit des Westens, trifft Müllers Anprangerung genau ins Schwarze: Auf Personen kommt es nach wie vor an, wenn man bestrebt ist, die Geschichte der SED/DDR zu beschreiben, umso mehr, wenn - wie im Folgenden - deren Verbindungen zu nicht sozialistischen Ländern den Hauptuntersuchungsgegenstand darstellen.

Viele der einschlägigen Akteure waren mir vor 2010, als ich eine internationale Konferenz über die Außenverbindungen der SED/DDR zu den „Bruderparteien“ Süd- und Westeuropas in der Bundesstiftung zur Aufarbeitung der SEDDiktatur in Berlin mit organisieren und leiten durfte, noch völlig unbekannt. Die Gespräche mit den Teilnehmenden bestärkten mich darin, den noch offenen Fragestellungen auf den Grund zu gehen. Der Co-Organisator jener Tagung, Prof. Dr. Arnd Bauerkämper, half mir maßgeblich dabei, den Blick auf Forschungskoordinaten der Verflechtungsgeschichte zu schärfen. Ihm bin ich dafür ebenso wie für die langjährige Zusammenarbeit zu besonderem Dank verpflichtet.

Die Forschungsarbeiten zur vorliegenden Studie begannen am Friedrich Meinecke Institut der Freien Universität Berlin und wurden u.a. durch eine generöse Förderung der Fritz Thyssen Stiftung - welche die Entstehung dieses Buchs ebenso ermöglicht hat - unterstützt. Die Fertigstellung der Monografie verdanke ich Prof. Dr. Wolfgang Mueller, unter dessen Federführung die Arbeit als Habilitationsschrift an der Universität Wien eingereicht und Ende November 2020 von deren historisch-kulturwissenschaftlicher Fakultät angenommen wurde. Den Mitgliedern der Jury, den Professor^innen Peter Becker, Johanna Gehmacher, Alojz Ivanišević, Kerstin Susanne Jobst, Claudia Kraft, Oliver Rothkolb und

1 Heiner Müller, Krieg ohne Schlacht. Leben in zwei Diktaturen, Köln 1992, S. 232. 
Philipp Ther - vor denen ich im Januar 2021 mit einem Vortrag meine Habilitation abgeschlossen habe - sowie den Gutachterinnen, Prof. Dr. Mario Keßler, Prof. Dr. Sara Lorenzini und Prof. Dr. Barbara Stelzl-Marx, möchte ich für alle Anregungen und Kritik ebenso meinen Dank aussprechen.

Die vielen Gespräche mit Kolleginnen und Kollegen haben der Genese der Studie bedeutende Impulse gegeben: $u$. a. Prof. Dr. Stefan Berger, Prof. Dr. Patrizia Dogliani, Prof. Dr. Mark Kramer, Prof. Dr. Ulrich Pfeil, Prof. Dr. Silvio Pons und Prof. Dr. Hermann Wentker bin ich dafür sehr verbunden.

Darüber hinaus kamen der Arbeit die wertvollen und akribischen Lektoratshinweise von Andrea Enders und Jan-Pieter Forßmann sehr zugute. Ihnen sowie allen fernen und nahen, hier nicht namentlich zu nennenden Freunden und Freundinnen, die in der letzten Dekade zu deren Genese beigetragen haben, schulde ich deshalb besondere Dankbarkeit.

Meinen Eltern, die mich unaufhaltsam fordern und in jeglicher Hinsicht fördern, ist dieses Buch gewidmet.

Nicht zuletzt sei meiner Frau, die mich mit sanfter Hand und ruhiger Entschlossenheit, mit schallender Liebe und unermüdlichem Beistand auch in schwierigen Zeiten unterstützt und erleuchtet hat, liebsten Dank ausgesprochen. Ohne Dich wäre dieses Buch nicht entstanden.

Berlin, Oktober 2021

Francesco Di Palma 